THE KURUME MEDICAL JOURNAL Vol. 14, No. 2, 1967.

\title{
A VECTORCARDIOGRAPHIC STUDY OF DIASTOLIC OVERLOAD OF THE LEFT VENTRICLE IN DOGS
}

\author{
TSUKASA FUKAMI \\ The Third Department of Internal Medicine, \\ Kurume University School of Medicine, \\ Kurume, Japan
}

(Received for publication July 15, 1967)

In 1952, Cabrera et al ${ }^{1)}$ 2) reported that in aortic regurgitation, diastolic overload of the left ventricle would have a different electrocardiographic manifestation than that produced by a systolic overload, as noted in aortic stenosis. They demonstrated that in cases of diastolic overload, increased voltage in the $\mathrm{Q}$ loop was manifested by displacement to the right and anterior; an increase in the voltage of the $\mathrm{R}$ loop was manifested by displacement to the left and posterior. It was also noted that in cases of systolic overload, the $\mathrm{Q}$ loop became smaller or even disappeared. The $\mathrm{R}$ loop voltage increased and became posteriorly displaced and the $\mathrm{S}$ loop disappeared or became straightened.

The purpose of this study is to observe the vectorcardiographic features in experimentally produced diastolic overloading of the left ventricle, and an attempt was made to search for a correlation between vectorcardiographic changas of diastolic overload and quantitative analysis of hypertrophy.

\section{MATERIAL AND METHODS}

Eighteen mongrel dogs weighing from 8 to $16 \mathrm{~kg}$ were anesthetized with sodium pentobarbital in the dosage of $25 \mathrm{mg}$ per $\mathrm{kg}$. Under spontaneous breathing of room air, the carotid artery and external vein were exposed and a $9 \mathrm{~F}$ Cournand catheter was inserted and the pressure curves of the left and right ventricles and aorta were recorded. Sanborn-poly-viso recorder model 67-1,200 was employed to allow simultaneous measurement of the pressure curves and the vectorcardiogram.

Improved straight grasping forceps were inserted to the aortic valves, a leaflet was extirpated or perforated satisfactorily without damaging the heart muscle. The confirmatory evidence of the resultant aortic insufficiency (AI) was noted by a marked fall of the aortic diastolic pressure with a typical murmur heard over base of the heart.

Mc Fee's corrected orthogonal lead system for dogs ${ }^{3)}$ was used for the electrocardiographic recording before and 10 weeks following the AI produced animal. 
Simultaneous recordings of scalar leads, $\mathrm{X}, \mathrm{Y}$ and $\mathrm{Z}$ were taken at a paper speed of $100 \mathrm{~mm} / \mathrm{sec}$. The polarity in the three orthogonal leads were used as follows: $\mathrm{X}$ axis, right negative, left positive $\mathrm{Y}$ axis, superior negative, inferior positive; $\mathrm{Z}$ axis, posterior negative, anterior positive.

The change of each instantaneous QRS complexes and the magnitude ( $\mathrm{mm}$ ), azimuth $\left(\mathrm{H}^{0}\right.$ degree) and elevation $\left(\mathrm{V}^{0}\right.$ degree) were calculated as follows :

$$
\begin{aligned}
& \text { 1. Magnitude }=\sqrt{\mathrm{X}^{2}+\mathrm{Y}^{2}+\mathrm{Z}^{2}} \\
& \text { 2. } \operatorname{Tan} \mathrm{H}^{0}=\frac{\mathrm{Z}}{\mathrm{X}} \\
& \text { 3. } \operatorname{Cos} \mathrm{V}^{0}=\frac{\mathrm{Y}}{\sqrt{\mathrm{X}^{2}+\mathrm{Y}^{2}+\mathrm{Z}^{2}}}
\end{aligned}
$$

Eight of 18 experiments, which the increased depth of the $Q$ waves were observed in lead $\mathrm{X}$, were selected at random for pathologic study.

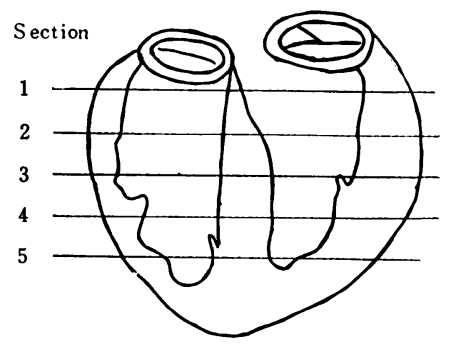

b

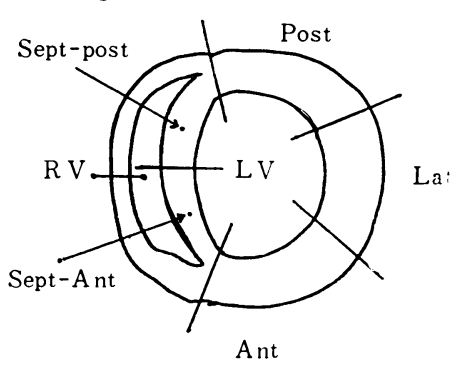

Fig. 1 .

As shown in Fig. 1, the heart was divided into six cross-sectional sections against the axis. Microscopic examination was performed with $\mathrm{HE}$ and sudan staining following formalin-fixation. The walls of left ventricle, including the ventricular septum, were separated into five portions. Each portion was divided into three parts ; epicardial, middle and endocardial regions ${ }^{4)}$. The extent of the hypertrophic alteration were expressed in the use of five groups and its weighting in accordance to severity was reflected in Table 14) $^{\text {5). }}$.

Hypertrophic alterations were classified into three categories, hypertrophic, degenerative and fibrous. According to Gore's ${ }^{5)}$ atherosclerotic profile, these alterations were considered to have a semilogarithemic relationship as to the 
TABLE 1

\begin{tabular}{clcc}
\hline Group & $\begin{array}{c}\text { Traditional } \\
\text { grade }\end{array}$ & $\begin{array}{c}\text { Proportion of hypertrophic } \\
\text { change }\end{array}$ & Weighting \\
\hline O & Negligible & Less than $5 \%$ & $\times 1$ \\
A & Minimal & $6-15 \%$ & $\times 4$ \\
B & Mild & $16-30 \%$ & $\times 10$ \\
C & Moderate & $31-50 \%$ & $\times 16$ \\
D & Severe & More than $50 \%$ & $\times 30$ \\
\hline
\end{tabular}

extent and grade of hypertrophy. As fibrous changes within the heart muscle were sorrounded by varying degrees of normal heart muscle, classification into four grades are shown in Table 2. For example, heart muscle with 15\% involvement of its normal tissue, would be classified as group A. With this degree of involvement and if previously ascertained that eight-tenth of the tissue was hypertrophied, one-tenth degenerated, and one-tenth demonstrating fibrosis (moderate), the occupied portion of the normal muscle, in a fractional area under facilitated visual estimation, would result in this hypertrophic profile being A $811^{\mathrm{m}}$ and the hypertrophic index may be derived by the following calculation:

$$
4\left(\frac{8}{10}+\frac{1}{10} \times 10+\frac{1}{10} \times 40\right)=23.2
$$

Since the theoretic range it from Oooo (o) to Do10 (3000) and for the convenience of indexing, $1 / 3$ is taken for calculation, thus the hypertrophic index becomes $23.2 \times 1 / 3=7.7$.

TABLE 2

\begin{tabular}{|c|c|c|c|}
\hline \multicolumn{2}{|c|}{ Grade } & \multirow{2}{*}{$\begin{array}{l}\text { Ratio of fibrosis against } \\
\text { normal heart muscle }\end{array}$} & \multirow{2}{*}{$\begin{array}{l}\text { Weighting } \\
\times 20\end{array}$} \\
\hline Mild & I & & \\
\hline Moderate & $\mathrm{m}$ & $21-50 \%$ & $\times 40$ \\
\hline Severe & $\mathrm{s}$ & $51-99 \%$ & $\times 70$ \\
\hline Complete & $\mathrm{c}$ & Completely fibrosis & $\times 100$ \\
\hline
\end{tabular}

\section{RESULTS}

1. QRS patterns of scalar electrocardiogram.

Fig. 2 shows the mean QRS patterns in three scalar leads before and after the production of the experimental aortic insufficiency (AI).

Lead X. Deflection of $\mathrm{Q}$ waves were significantly increased at 0.005 sec. $(\mathrm{p}<$ $0.01)$ and at 0.010 sec. $(\mathrm{p}<0.005)$. Rx denotes almost no change at 0.015 sec. and 0.020 sec. At 0.025 sec., Qx showed a marked increase in voltage, which was statistically significant when compared with the normal $(p<0.005)$. At 0.040 sec., $\mathrm{S}$ waves were noticed before AI disappeared.

Lead $\mathrm{Y}$. The $\mathrm{Q}$ waves were not seen in this lead and no significant changes were noted at 0.010 and 0.020 sec. At 0.025 sec., there were pronounced differences 


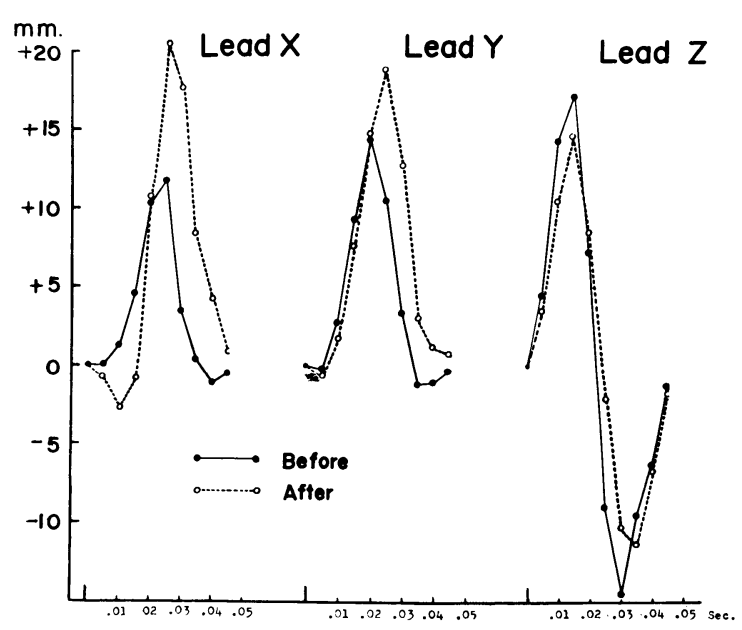

Fig. 2. Mean patterns in three orthogonal leads before and after experimental aortic insufficiency.

in voltage after chronic AI as compared with the normal $(\mathrm{p}<0.01)$.

Lead Z. In both groups, there were no significant differences.

2. Changes of instantaneous spatial vectors.

Table 3 and Fig. 3, 4, 5 show the mean values of the azimuth, elevation and magnitude of instantaneous vectors.

TABLE 3

Mean values of azimuth, elevation and magnitude of $i$-nstantaneous vectors before and after experimental astic insufficiency

\begin{tabular}{|c|c|c|c|c|c|c|c|c|c|c|c|}
\hline & & & 0.005 & 0.010 & 0.015 & 0.020 & 0.025 & 0.030 & 0.035 & 0.040 & 0.045 \\
\hline \multirow{5}{*}{ Azimuth } & \multirow{2}{*}{ Before } & Mean & 95.6 & 83.2 & 74.5 & 36.6 & -36.9 & -76.7 & -91.3 & -108.2 & -131.0 \\
\hline & & S. D. & 18. 1 & 15.4 & 19. 3 & 31.7 & 28.7 & 22.6 & 15.0 & 33.0 & 27.0 \\
\hline & \multirow{2}{*}{ After } & Mean & 118.8 & 106. 9 & 84.2 & 36.2 & -10.1 & -40.1 & -67.6 & -76.1 & 75.8 \\
\hline & & S. D. & 27. 0 & 26.8 & 34.2 & 29.2 & 25.5 & 22.7 & 37.9 & 41. 0 & 33. 6 \\
\hline & $\mathrm{P}$ value & & \multicolumn{2}{|c|}{$\mathrm{P}<0.005 \mathrm{P}<0.005$} & & & \multicolumn{3}{|c|}{$\mathrm{P}<0.025 \mathrm{P}<0.005 \mathrm{P}<0.025$} & & \\
\hline \multirow{5}{*}{ Elevation } & \multirow{2}{*}{ Before } & Mean & 100.6 & 84.0 & 65.8 & 45.8 & 54.8 & 77.6 & 101.6 & 107. 6 & 97.3 \\
\hline & & S. D. & 25.7 & 20.8 & 14. 1 & 18.0 & 14.6 & 11.8 & 25.0 & 23.8 & 28.0 \\
\hline & \multirow{2}{*}{ After } & Mean & 105.7 & 82.4 & 65.0 & 45.7 & 48. 3 & 68.6 & 86.9 & 95.0 & 100.8 \\
\hline & & S. D. & 21.7 & 17. 4 & 25.0 & 17.5 & 9.7 & 18.9 & 29.2 & 21.0 & 32.8 \\
\hline & $P$ value & & & & & & & $\mathrm{P}<0.01$ & $\mathrm{P}<0.01$ & & \\
\hline \multirow{5}{*}{$\underset{\text { tude }}{\text { Magni- }}$} & \multirow{2}{*}{ Before } & Mean & 4.5 & 16.1 & 19. 1 & 21.3 & 20.1 & 15. 7 & 11.2 & 5. 2 & 2. 2 \\
\hline & & S. D. & 3. 6 & 9.7 & 7. 0 & 7. 7 & 6.5 & 5.0 & 7. 3 & 3.4 & 2. 3 \\
\hline & \multirow{2}{*}{ After } & Mean & 4. 2 & 12.1 & 17. 3 & 22.1 & 30.3 & 27.9 & 18.4 & 10.6 & 3.7 \\
\hline & & S. D. & 3.1 & 5.5 & 5.4 & 6.6 & 8. 6 & 11.0 & 11. 3 & 8.4 & 3. 6 \\
\hline & $P$ value & & & & & & $\mathrm{P}<0.00$ & $\mathrm{P}<0.005$ & $\mathrm{P}<0.025$ & & \\
\hline
\end{tabular}


Azimuth

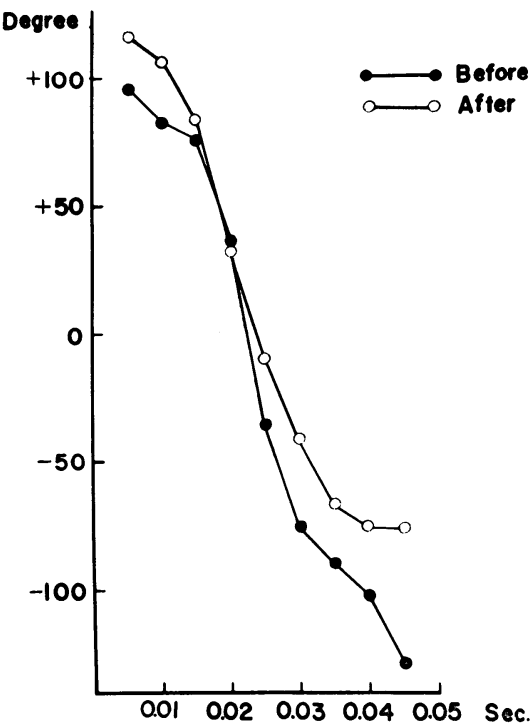

Fig. 3. Changes of azimuth of instantaneous vectors before and after experimental aortic insufficiency.
Elevation

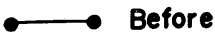

After

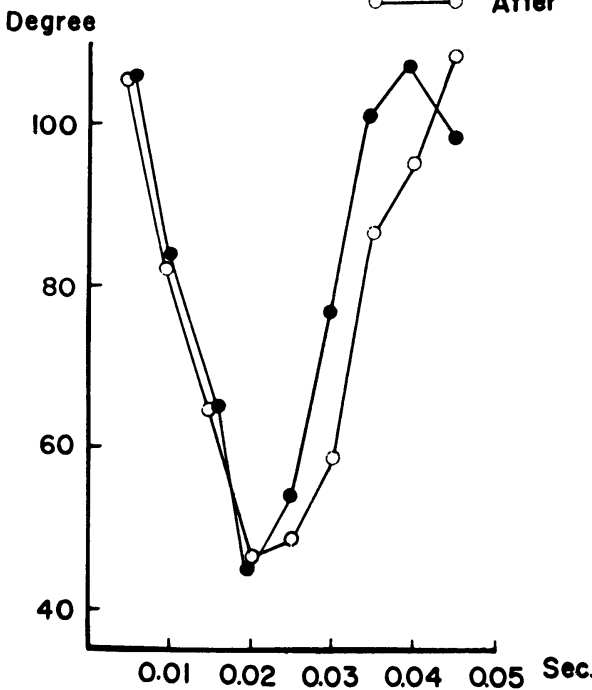

Fig. 4. Changes of elevation of instantaneous vectors before and after experimental aortic insufficiency.

Magnitude

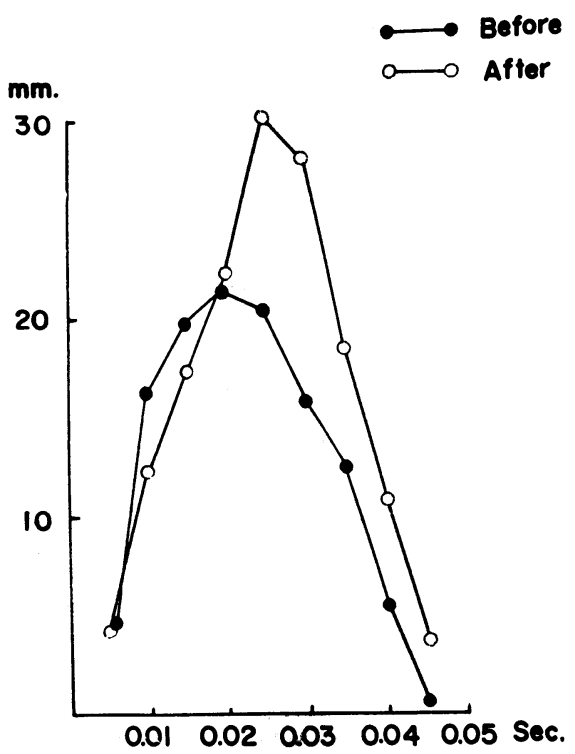

Fig. 5. Changes of magnitude of instantaneous vectors before and after experimental aortic insufficiency. 
0.005 Sec. Vector: The azimuth was considerably increased, however, there was little difference in elevation. The strength was increased after AI as compared with the normal, the vectors being directed to the right anterior part.

0.010 Sec. Vector: The spatial vectors showed a significant difference in deviation when compared with the normal. The vectors in this second were quite characteristic, right and anterior components being prominent.

0.020 Sec. Vector: The azimuth and the elevation were not statistically significant between the before and after AI production.

0.025 Sec. Vector: The azimuth deviated posteriorly while the vector was more posteriorly oriented. It can be seen that the magnitude of this second was markedly increased and was significantly different from that of normal $(p<0.005)$.

0.030 Sec. Vector: The spatial vectors were mostly deviated to left posterior.

0. 035 Sec. Vector: All vectors were located in the lower left posterior part and were found to be less valuable.

$0.040,0.045$ Sec. Vector: These spatial vectors were scattered widely and no significant differences were noted.

3. QRS duration and $\mathrm{Rx}$ peak time.

The results of the measurement of the QRS duration and $\mathrm{Rx}$ peak time are shown in Table 4. The mean times of the normal were $0.046 \pm 0.003$ sec. $0.025 \pm$ 0.003 on the average for QRS-duration and $\mathrm{Rx}$ peak times and prolonged to $0.050 \pm 0.003$ sec. $(p<0.01), 0.025 \pm 0.003$ sec. $(p<0.025)$ respectively after aortic insufficiency.

TABLE 4

Changes of mean QRS duration and $R x$ peak time

before and after experimental astic insufficiency

\begin{tabular}{|c|c|c|}
\hline & $\begin{array}{r}\text { QRS-duration } \\
\text { (sec.) }\end{array}$ & $\begin{array}{r}\text { Rx Peak Time } \\
\text { (sec.) }\end{array}$ \\
\hline Before & $0.046 \pm 0.003$ & $0.022 \pm 0.003$ \\
\hline After & $0.050 \pm 0.003$ & $0.025 \pm 0.003$ \\
\hline$P$ value & $\mathrm{P}<0.01$ & $\mathrm{P}<0.025$ \\
\hline
\end{tabular}

4. QRS loop.

As can be seen in Fig. 6, the mean QRS loops in the frontal and horizontal planes were characteristically different to AI than those noted before the procedure.

Frontal plane: Seven of the 16 loops showed a counterclockwise (CCW) direction, five showed a clockwise (CW) direction, and four a "figure 8" type.

After AI, seven configuration loops showed a CCW direction, seven loops demonstrated the "figure 8 " type of loop. Two were CW.

Horizontal plane: Normally all cases were CCW loop while three "figure 8" type loops were noted after AI production.

Sagittal plane: All showed CW loops and no significant alterations were observed after AI. 


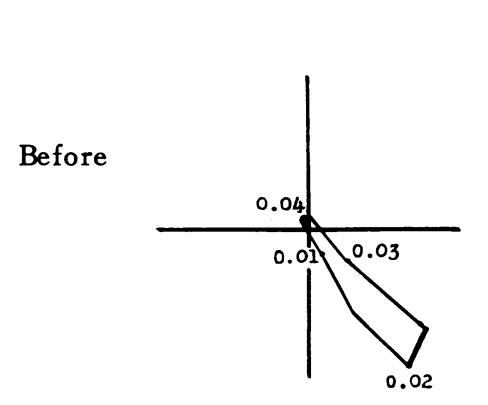

Frontal

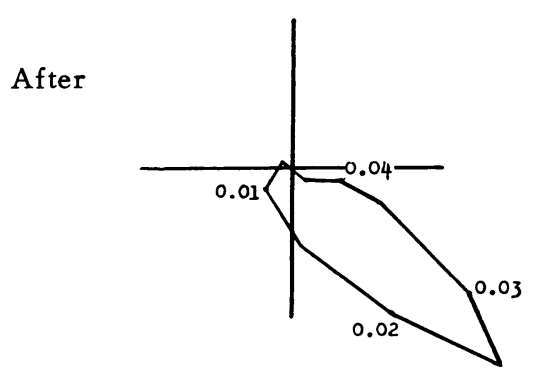

Mean QRS loop

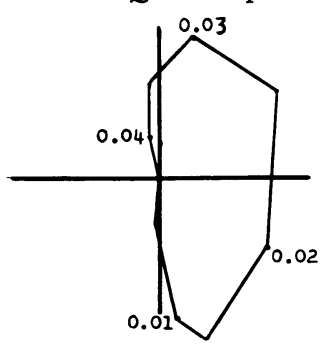

Horizontal

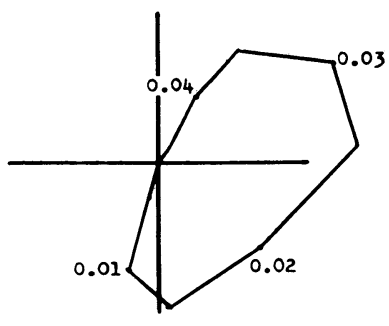

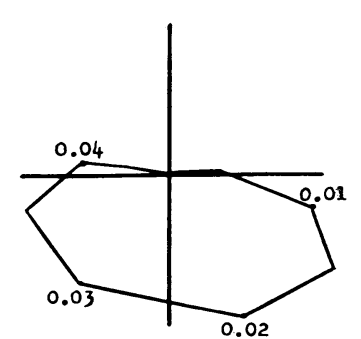

Sagittal

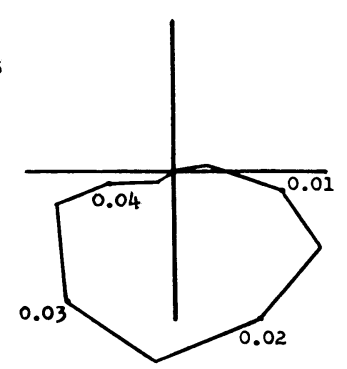

Fig. 6. Changes of mean QRS loops before and after experimental aortic insufficiency.

TABLE 5

Distribution of mean values of hypertrophic index End: Endocardial part. $\quad$ M. : Middle part. $\quad$ Ep. : Epicardial part. $P$ : Papillary musccle. $\quad R V-S:$ Right ventricle side.

\begin{tabular}{|c|c|c|c|c|c|c|c|c|c|c|c|c|c|c|c|c|c|c|}
\hline \multirow[b]{2}{*}{ No. } & \multicolumn{4}{|c|}{ Anterior } & \multicolumn{4}{|c|}{ Lateral } & \multicolumn{4}{|c|}{ Posterior } & \multicolumn{3}{|c|}{ Ant. Septum } & \multicolumn{3}{|c|}{ Post. Septum } \\
\hline & End & M. & EP. & P. & End & M. & EP. & P. & End & M. & EP. & P. & End & M. $\left|\begin{array}{c}\mathrm{RV} \\
-\mathrm{S}\end{array}\right|$ & P. & End & M. $\left|\begin{array}{c}\mathrm{RV} \\
-\mathrm{S}\end{array}\right|$ & P. \\
\hline 2036 & 8. 3 & 10.8 & 3.44 & 46. 0 & 12.9 & 3.1 & 6. 9 & 37.01 & 16.8 & 6. 0 & 6.62 & 24.9 & 12.5 & 3. 614.2 & 67.2 & $15.8^{\prime}$ & 6.520 .3 & 57.1 \\
\hline 4057 & 11.0 & 3.8 & 4. 0 & 16.5 & 8.8 & 3.0 & 1. 9 & 20.5 & 10.1 & 6.7 & 7.63 & 31.8 & 10.8 & 4. 13.8 & 23.6 & 11.7 & 4.38 .7 & 38.1 \\
\hline 5066 & 7.2 & 6.0 & 4.53 & 37.0 & 25.6 & 8.6 & 9.1 & 37.6 & 14.3 & 9.1 & 7.43 & 33.4 & 8.1 & 1.88 .3 & 37.7 & 34.8 & $\begin{array}{ll}4.5 & 6.5\end{array}$ & 44.5 \\
\hline 5069 & 7.1 & 1. 7 & 2.19 & 94.5 & 10.5 & 0.9 & 8. 6 & 89.0 & 11.1 & 3. 9 & 4.35 & 50.9 & 2.8 & 1.6 5.4 & 36.4 & 11.4 & $\begin{array}{ll}3.5 & 6.9\end{array}$ & 2.5 \\
\hline 5089 & 22.2 & 8. 8 & 5.32 & 25.4 & 18.41 & 10.5 & 12.0 & 188.5 & 13.9 & 7.2 & 7.5 & 19. 8 & 17. 7 & 9. 212.2 & 63.2 & 17.8 & 4.913 .4 & 52.0 \\
\hline 8086 & 13. 9 & 1.5 & 8.35 & 58.5 & 12.4 & 1.3 & 7. 3 & 29. 7 & 12.4 & 2.5 & 7.31 & 12.6 & 2.4 & 1.33 .6 & 14.9 & 1. 3 & 1.321 .63 & 33. 2 \\
\hline Average & 11.6 & 5.4 & 4. 6 & 46.3 & 14.8 & 4.5 & 7.5 & 67.1 & 13. 1 & 5. 9 & 6.82 & 28.9 & 9.0 & 3. 67.9 & 40.9 & 15.4 & 4. 212.93 & 37.9 \\
\hline \multicolumn{19}{|l|}{ Control } \\
\hline 9057 & 4. 6 & 1.1 & 1. 8 & 7.1 & 4.7 & 0.9 & 2.3 & 9.7 & 4.5 & 1. 3 & 1.9 & 9.7 & 3. 9 & 1. 32.0 & 6.3 & 6.8 & 1. 3 & 9. 0 \\
\hline 9058 & 3. 9 & 1.9 & 1.8 & 5.3 & 3.3 & 1.3 & 1.3 & 8.0 & 4.5 & 1. 3 & 1.3 & 8. 1 & 3.3 & 1. 3 3. 2 & 4.0 & 4.1 & $\begin{array}{lll}1.3 & 2.9\end{array}$ & 6.4 \\
\hline Average & 4. 2 & 1.5 & 1. 8 & 6.2 & 4.0 & 1.1 & 1.8 & 8.9 & 4.5 & 1. 3 & 1.6 & 8. 9 & 3. 6 & 1. $3 \quad 2.6$ & 5.2 & 5.4 & 1. 3 2. 2 & 7. 7 \\
\hline
\end{tabular}

5. Pathologic findings.

Table 5 presents the mean value of the hypertrophic index in each fractional area. In the heart muscle with chronic aortic insufficiency, the hypertrophic index was higher in the posterior septum showing 15.4, this was followed by the 
lateral with 14.8. All cases demonstrated considerable dilatation of the heart as compared to the normal, demonstrating the mechanical influences of hemodynamics prominent in the papillary muscle. In general, the hypertrophic index was higher in cases with chronic AI, as compared to those of AS.

As shown in Table 6, hypertrophic changes were increased in section 1 and section 4 of the cross-sectional heart.

TABLE 6

Mean values of hypertrophic index in cross-sectional sections

\begin{tabular}{c|r|r|r|r|r}
\hline Dog No. & Section 1 & Section 2 & Section 3 & Section 4 & Section 5 \\
\hline 2036 & 9.2 & 9.1 & 10.6 & 10.2 & 8.8 \\
4057 & 7.6 & 6.1 & 8.3 & 8.2 & 3.8 \\
5066 & 14.0 & 7.8 & 12.7 & 13.9 & 7.4 \\
5069 & 10.9 & 4.0 & 4.2 & 5.3 & 2.9 \\
5089 & 11.9 & 16.5 & 11.5 & 14.4 & 7.3 \\
8086 & 7.0 & 8.6 & 5.4 & 5.4 & 7.0 \\
Average & 10.0 & 8.7 & 8.8 & 9.6 & 6.2 \\
\hline Control & & & & & \\
\hline 9057 & 2.6 & 2.7 & 2.3 & 2.7 & 1.7 \\
9058 & 2.1 & 2.5 & 2.3 & 2.9 & 2.0 \\
Average & 2.4 & 2.6 & 2.3 & 2.8 & 1.8 \\
\hline
\end{tabular}

\section{DISCUSSION}

The interesting concept of systolic and diastolic overloading of the heart was introduced by Cabrera and Monroy ${ }^{\prime \prime}$. They stated that in systolic overloading of the left ventricle, as seen in aortic stenosis and systemic hypertension, the electrocardiographic pattern is characterized by ST segment depression and $\mathrm{T}$ wave inversion in the left ventricular leads. The heart demonstrates a thick-walled left ventricle without significant dilatation of the cavity as seen at autopsy.

On the contrary, in the case with diastolic overloading of the left ventricle, the electrocardiographic pattern is characterized by tall $R$ waves with late peaks in the left precordial leads, deep $S$ waves in the right precordial leads and tall peaked $\mathrm{T}$ waves with upward ST displacement in left precordial leads. At autopsy there usually is considerable dilatation of the left ventricular cavity. Vectorcardiographically, an augmentation of the $\mathrm{Q}$ loops oriented to the right and anteriorly was one of the characteristical findings in diastolic overload of the left ventricle, whereas a reduction in or absence of the $Q$ loops was a sign of systolic overload. Although this concept has been a unique approach in the diagnosis of certain congenital and acquired heart disease lesions, it has not been generally accepted because of the lack of evidence correlating the anatomic and electrocardiographic findings ${ }^{7)}{ }^{8)}$.

In the observations presented by Selzer ${ }^{7)}$, he demonstrated that in left 
ventricular hypertrophy the electrocardiographic abnormalities due to systolic overload were different from those due to diastolic overload. Sedziwy and Shillingford ${ }^{8)}$ demonstrated considerable overlap in their cadiographic patterns of the two groups. They also stated it had not been possible to clearly separate them in clinical practice. Similar findings were reported by Yano and Pipberger ${ }^{9)}$.

These discrepancies are considered to be variations in the clinical complexities and various aspects of hemodynamics. Hence an attempt to formulate a study of vectorcardiographic changes in purely induced aortic insufficiency was attempted, and the relationship to the pathologic findings were studied.

It is generally agreed that the initial forces of the vectors, which constitute the $\mathrm{Q}$ loops, results from left to right septal activation. Recently, Kimura and Toshima ${ }^{10}{ }^{11)}$ clinically illustrated that in cases of aortic insufficiency the initial vectors deviated more to the right anterior part and differed from cases having aortic stenosis where the initial spatial vectors deviated to left anterior.

Deviation of initial spatial vectors from the right anterior region to the left anteriorly region according to related development of the hypertrophic results from experimental AS as was demonstrated by $\mathrm{Abe}^{6)}$.

In this study, displacement of initial forces that were statistically significant between AI and AS were characteristical sign of diastolic overloading of the left ventricle which may be useful in diagnostic consideration for its use in practice.

Increased magnitude, or high voltage of the complex (QRS) has been known to be the most common electrocardiographic sign of left ventricular overloading in orthogonal, as well as in the twelve-lead electrocardiogram, and has been attributed to the delay in the transmyocardial excitation of the thickened muscular walls ${ }^{11}{ }^{12)}$. With this study a marked increase in magnitude or high voltage in lead X was observed. These observations were more prominent than those noted with lesions of $\mathrm{AS}^{6)}$.

The prolongation of the QRS interval and the prolonged time interval between the onset of the QRS complex and the peak of the $\mathrm{R}$ wave in lead X, as observed in left precordial leads, are commonly encountered abnormalities associated with left ventricular overloading. As the severity of hypertrophied heart advance, the prolonged QRS duration denotes a $\mathrm{LBBB}^{13)}$. Yano and Pipberger ${ }^{9)}$ have reported that the Rx peak time was more useful in the diagnosis of hypertrophic changes than the QRS duration. Solof $\mathrm{f}^{14)}$ has indicated the importance of the availability of the QRS duration in clinical practice. The prolongation of the QRS duration and $\mathrm{Rx}$ peak time were observed in pure diastolic overloading of the heart and these were considered to result from the delay in the transmyocardial excitation wave as caused by increasing muscle mass and the dilatation of the ventricular walls ${ }^{13)}$. An increasing depth of the $Q$ waves in the left precordial lead and the deviation of the initial spatial vectors to the right anterior region have been attributed to hypertrophy of the interventricular septum with an increase in the electromotive forces appearing during its activation from the left to the right 11) 13). 
In cases of $\mathrm{AS}$, the $\mathrm{Q}$ loops became smaller or disappeared ${ }^{2)}{ }^{6)}{ }^{15)}$. Hugenholtz and Gamboa ${ }^{16)}$ have demonstrated that in congenital aortic stenosis, the magnitude of the initial vector was decreased. A significant increase in the depth of the $\mathrm{Q}$ waves in lead $\mathrm{X}$, as noted in this experiment, is associated with the additional magnitude and prolongation of the QRS interval. This finding demonstrates the correlation between these alterations and pathological findings suggesting that the increasing magnitude of the $Q$ waves and the displacement of the initial spatial vectors are presumably only partially resultant from the hypertrophy of the posterior septum. This explanation seems to be adequate for the findings of AI but not for those of the AS group ${ }^{6)}$. In comparing AI and AS, there appears to be no significant difference in the hypertrophic index of each section of the heart.

A resonable explanation of the direction and magnitude of the initial vectors in AI could be as follows: The dilatation of the left ventricle that results from aortic regurgitation could lead to a relative delay in the activation of the free wall of the left ventricle without cancellation of the spatial forces.

\section{SUMMARY}

A vectorcardiographic study using McFee's orthogonal lead system was performed in 18 mongrel dogs before and after experimentally produced aortic insufficiency.

In 8 experiments, the correlation between vectorcardiographic alterations and the pathologic evidence of diastolic overloading of left ventricle were studied.

The initial spatial vectors deviated to more right anteriorly after 10 weeks experimental AI as compared with the before noted procedure. There was also a significant difference in the magnitude at 0.025 seconds. The prolongation of the $\mathrm{QRS}$ duration and the Px peak time were detected after producing AI. Compared with AS, the hypertrophic index in AI was generally extended and became prominent in the endocardial regions. The explanation of the deviation of the initial vectors is demonstrated by a relative delay in the activation of the free wall of the left vantricle with noncancallation of the septal force.

\section{ACKNOWLEDGMENT}

The author wishes to express his appreciation to Prof. Noboru Kimura and Associate Prof. Hironori Toshima for their helpful suggestions throughout the course of this work and in reviewing the manuscript. Dr. Y. Nakaura and Dr. Abe have also contributed greatly to the success of this experiment. 


\section{REFERENCES}

1) Cabrera, E. and Monroy, J. R. : Systolic and diastolic over loading of the heart, I. Amer. Heart J., 43, 661, 1952. II. Amer. Heart J, 43, 669, 1952.

2 ) Cabrera, E. and Gaxiola, A. : Diagnostic contribution of the vectorcardiogram in hemodynamic overloading of the heart. Amer. Heart J, 60, 296, 1960.

3) McFEe, R. : An orthogonal lead system for clinical electrocardiography. Amer. Heart J, 62, 93, 1961.

4) Nakashima, T. and NAKaura, Y. : A quantitative analysis of hypertrophy of the heart muscle. Inpress.

5 ) Gore, L. and TejadA, C. : The quantitative appraisal of atherosclerosis. Amer. J. Path., 33, 875, 1957.

6 ) ABE, K. : A study on vectorcardiogram of experimental aortic stenosis. J. Kurume Medical Association, 29, 1115, 1966.

7 ) Selzer, A. et al. : Electrocardiographic findings in concentric and eccentric left ventricular hypertrophy. Amer. Heart J., 63, 320, 1962.

8 ) Sedziqy, L. and Shillngford, J. : Cardiographic patterns in systolic and diastolic overload of the left ventricle. British Heart J, 23, 533, 1962.

9) Yano, K. and Pipberger, V.H. : Correlations between radiologic heart size and orthogonal electrocardiograms in patient with left ventricular overloading. Amer. Heart J., 67, 44, 1964.

10) Kimura, N. and Toshima, H. : Progress in clinical electrocardiography, electrocardiograms in left and right ventricular hypertrophy. Naika, 16, 440, 1965.

11) Sodi-PAllares, D. : New basis of electrocardiography. The C. V. Mosby Company, St. Louis, 1959.

12) Scott, R. C.: The correlation between the electrocardiographic patterns of ventricular hypertrophy and the anatomic findings. Circulation, 21, 256, 1960.

13) Scott, R. C.: The electrocardiographic diagnosis of left ventricular hypertrophy in the presence of left bundle branch block. J. Lob. \& Clin. Med., 50, 953, 1957.

14) Soloff, A. and LAwrence, J. W. : The electrocardiographic findings in left ventricular hypertrophy and dilatation. Circulation, 26, 553, 1962.

15) Toshima, H. et al. : Vectorcardiographic studies in acquired valvular disease with reference to the diagnosis of left ventricular hypertrophy. Circulation, 35, 132, 1967.

16) Hugenholtz, P. G. and Gamboa, R. : Effect of chronically increased ventricular pressure on electrical forces of the heart: correlation between hemodynamic and vectorcardiographic data (Frank System) in 90 patients with aortic or pulmonic stenosis. Circulation, 30, $511,1964$. 